\title{
Cortical Thickness, Brain Metabolic Activity, and In Vivo Amyloid Deposition in Asymptomatic, Middle-Aged Offspring of Patients with Late-Onset Alzheimer's Disease.
}

Bárbara Duarte-Abritta, B.Sc. ${ }^{1 *}$, Mirta F. Villarreal, Ph.D. ${ }^{1-3^{*}}$, Carolina Abulafia, B.A. ${ }^{1,2,5}$, David Loewenstein, Ph.D. ${ }^{6}$, Rosie E. Curiel Cid, Psy.D. ${ }^{6}$, Mariana N. Castro, M.D., Ph.D. ${ }^{1,2,4}$, Ezequiel Surace, Ph.D. ${ }^{2,7}$, Stella-Maris Sánchez, B.Sc. 1,2,3, Daniel E. Vigo. ${ }^{2,5}$, Silvia Vázquez, M.D. ${ }^{8}$, Charles B. Nemeroff, M.D., Ph.D. ${ }^{6}$, Gustavo Sevlever, M.D., Ph.D. ${ }^{9}$, Salvador M. Guinjoan, M.D., Ph.D. ${ }^{1,2,4,10}$.

${ }^{1}$ Service of Psychiatry, FLENI Foundation, Buenos Aires, Argentina

${ }^{2}$ National Council for Scientific and Technological Research (CONICET), Argentina

${ }^{3}$ Department of Physics, University of Buenos Aires School of Exact and Natural sciences, Argentina.

${ }^{4}$ Department of Psychiatry and Mental Health, University of Buenos Aires School of Medicine, Argentina.

${ }^{5}$ Institute for Biomedical Research (BIOMED), School of Medical Sciences, Universidad Católica Argentina, Buenos Aires, Argentina. 
${ }^{6}$ Department of Psychiatry and Behavioral Sciences, Miller School of Medicine, University of Miami, United States of America.

${ }^{7}$ Department of Neuropathology \& Molecular Biology, Fleni Foundation, Buenos Aires, Argentina.

${ }^{8}$ Center for Molecular Imaging, Fleni Foundation, Buenos Aires, Argentina.

${ }^{9}$ Departmentof Neuropathology \& Molecular Biology, Fleni Foundation, Buenos Aires, Argentina.

${ }^{10}$ Neurophysiology I, University of Buenos Aires School of Psychology

*Both authors contributed equally to this paper.

\section{Corresponding author}

Salvador M. Guinjoan

Service of Psychiatry, Fleni Foundation

Montañeses $23255^{\text {th }}$ floor

C1428AQK Buenos Aires

Argentina

sguinjoan@fleni.org.ar

+541157773200 x2514/2531 


\section{Abstract}

The natural history of preclinical late-onset Alzheimer's disease (LOAD) remains obscure and has received less attention than that of early-onset AD (EOAD), in spite of accounting for more than $99 \%$ of cases of AD. With the purpose of detecting early structural and functional traits associated with the disorder, we sought to characterize cortical thickness and subcortical grey matter volume, cerebral metabolism, and amyloid deposition in persons at risk for LOAD in comparison with a similar group without family history of AD. We obtained 3T T1 images for gray matter volume, FDG-PET to evaluate regional cerebral metabolism, and PET-PiB to detect fibrillar amyloid deposition in 30 middle-aged, asymptomatic, cognitively normal individuals with one parent diagnosed with LOAD (O-LOAD), and 25 comparable controls (CS) without family history of neurodegenerative disorders (CS). We observed isocortical thinning in AD-relevant areas including posterior cingulate, precuneus, and areas of the prefrontal and temporoparietal cortex in O-LOAD. Unexpectedly, this group displayed increased cerebral metabolism, in some cases overlapping with the areas of cortical thinning, and no differences in bilateral hippocampal volume and hippocampal metabolism. Given the importance of age in this sample of individuals presumably developing early AD-related changes, we controlled results for age and observed that most differences in cortical thickness and metabolism became nonsignificant; however, greater deposition of $\beta$-amyloid was observed in the right hemisphere including temporoparietal cortex, postcentral gyrus, fusiform inferior and middle temporal and lingual gyri. If replicated, the present observations of morphological, metabolic, 
and amyloid changes in cognitively normal persons with family history of LOAD may bear important implications for the definition of very early phenotypes of this disorder.

Keywords: Alzheimer's Disease, Cortical Thickness, FDG-PET, PET-PiB, ApoE4. 


\section{Introduction}

The pathophysiology of late-onset Alzheimer's Disease (LOAD), accounting for over $99 \%$ of cases of $A D$, is poorly understood and is an active area of investigation. Available data suggests AD probably results from a more complex mechanism than the early-onset form described by Alzheimer (1) and named after him by Kraepelin (2), even though these two clinical forms share the neuropathological landmarks of amyloid plaques, neurofibrillary tangles, and neurodegeneration. According to the dominant amyloid cascade hypothesis $(A C H)$, extrafibrillar amyloid deposition is followed by intracellular Tau-mediated toxicity and then neurodegeneration (3); however, a series of findings and the repeated failures of experimental agents targeting amyloid synthesis, deposition or clearance to treat Alzheimer's disease (AD) manifestations have questioned the heuristic validity of this theory $(4,5)$. It is relevant to note that despite the higher prevalence of LOAD, there is a paucity of studies exploring the early pathological processes of LOAD in individuals who are at-risk for this form of the disorder. Apart from aging, having a parent with LOAD is the main known risk factor $(6,7)$. This is partially explained by being a carrier of the APOE\&4 allele; however, given that many patients who develop LOAD are not APOE $\varepsilon 4$ allele carriers and many individuals who are hetero- or homozygous for APOE \&4 never develop LOAD.

Several studies have suggested three main findings associated with early LOAD pathophysiology. First, that there is a "dose effect" associated with heritable risk for LOAD biomarker changes prior to symptom onset. That is, maternal or biparental 
inheritance appears to confer more risk than paternal inheritance (8). Second, that familial risk is in general, associated with decreased brain metabolism, especially in AD-relevant areas such as medial temporal lobe, precuneus, and parietal cortex $(9,10)$. Third, that amyloid deposition and brain hypometabolism precede structural gray matter changes $(11,8)$. Another source of evidence for preclinical biomarkers of LOAD comes from studies of APOE $\varepsilon 4$ carrier status effects in populations of individuals with family history of LOAD, which is associated with early decreased brain metabolism, much like clinical forms of LOAD such as amnestic mild cognitive impairment and $A D$ dementia $(12,11)$. There is no uniform agreement in these studies, as for example, Mosconi et al. (8) did not find a significant contribution of $A P O E$ genotype in their sample of at-risk individuals. Perhaps similar to several other prevalent medical disorders with definite heritable risk but unknown risk genes and only partial concordance in monozygotic twins, various undetermined environmental factors probably interact with the vulnerability gene variants to contribute to the appearance of clinical forms of LOAD (6). Understanding that an as-yet unknown number of genes can contribute to LOAD, the chances of sampling the same gene subsets in different studies of limited sample size is quite low; thus, in the present study, we sought to characterize the presence of AD-related neuroimaging biomarkers in individuals with at least one parent affected with $\operatorname{LOAD}(13,14)$, in a sample of middle-aged, asymptomatic individuals in the Buenos Aires metropolitan area of Argentina. Peculiar characteristics of this sample include its ethnic and cultural homogeneity (all participants are Caucasian Argentine Hispanics), a relatively high level of formal education, the lack of significant comorbid cerebrovascular disease, and distinct 
dietary habits, including one of the highest red meat intake per person in the world, of approximately $40 \mathrm{~kg}$ per person per year on average (second only to that of neighboring Uruguay), which has been considered a risk factor for dementia in old age (15).

We hypothesized that these epidemiological peculiarities would not influence the presence of early biomarkers for this at-risk sample and, according to the $\mathrm{ACH}$, we predicted that in vivo evidence of amyloid deposition and hypometabolism in ADrelevant brain regions would precede, or at least be greater than, structural deficits as evaluated by subcortical structure volumes and isocortical thickness. We also predicted that the latter, if at all present at this early period of risk, would be present, or be greater, in mesial temporal structures including the hippocampus, than in neocortical regions.

\section{Materials and Methods}

\section{Sample}

A functional 18F-FDG PET image, an 11C-PIB-PET image of cerebral $\beta$ amyloid deposit and a structural T1-weighted MRI image were obtained in 30 LOAD offspring (O-LOAD) and 25 control subjects (CS) comparable in gender, age, and education level. The inclusion criteria for O-LOAD were as follows: 1) At least one parent diagnosed with probable LOAD according to the DSM-5 criteria, 2) 40-65 years old at the time of recruitment, and 3) $>7$ years of formal education. All participants were asked for clinical information of affected family members. For 
individuals who had received no treatment at the FLENI Foundation $(n=5)$ the parents' diagnosis of LOAD was confirmed by a clinician. In addition to clinical confirmation of LOAD in the parents, structural MRIs were available to confirm atrophic changes suggestive of $A D$ and absence of significant vascular disease in the parents of 15 participants. Of these, three had a positive PET-PiB test. The CS group had the same inclusion criteria except no family history of $A D$ or other neurodegenerative brain disease. The exclusion criteria for O-LOAD and CS were as follows: 1) Mini Mental State Examination (MMSE) score $<25,2$ ) compromised intellectual level based upon low levels of education and occupational history, 3) evidence of current progressive neurologic disease likely to impact cognitive performance, 4) history of substance abuse (alcohol, marijuana, stimulants, benzodiazepines, or other drugs), and 5) Hachinski score $>7$, to identify vascularderived cognitive impairment.

The study protocol was performed in accordance with the Declaration of Helsinki, and approved by the Bioethics Committee of the FLENI Foundation, Argentina. All participants provided their written informed consent for the study.

\section{T1 Image Acquisition}

MRI images were acquired on a 3 T GE Signa HDxt MRI machine with an eightchannel head coil. A high resolution T1 3D fast SPGR-IR image was acquired. One hundred and sixty-six sagittal contiguous slices were obtained in an acquisition matrix of $256 \times 256, \mathrm{TR}=7.256 \mathrm{~ms}, \mathrm{TE}=2.988 \mathrm{~ms}$, flip angle $8^{\circ}, \mathrm{FOV}=26 \mathrm{~cm}$, and slice thickness $=1.2 \mathrm{~mm}$. 


\section{Cortical and subcortical T1 Image Processing}

Cortical reconstruction and volumetric segmentation were performed with the FreeSurfer image analysis program version Linux-centos6_x86_64-stable-v6-beta20151015 , which is available for online download at (http://surfer.nmr.mgh.harvard.edu/). The FreeSurfer pipeline removes non-brain tissue using a hybrid watershed/surface deformation procedure (16), automated Talairach transformation, segmentation of the subcortical white matter (WM) and deep gray matter (GM) volumetric structures $(17,18)$, intensity normalization (19), tessellation of the GM/WM boundary, automated topology correction $(20,21)$, and surface deformation following intensity gradients to optimally place the GMNM and $\mathrm{GM} /$ cerebrospinal-fluid borders at the location where the greatest shift in intensity defines the transition to the other tissue class $(22,23,24)$.

The morphometric evaluation of each hemisphere was performed independently. Each volume and surface obtained was carefully reviewed by a two investigators blind to the participant's study condition (BDA, SMG) and edited manually by one of these investigators (BDA) as necessary to conform to the anatomically determined limits. Major topological inaccuracies were corrected with vertex edits or control points. Finally, surface maps were smoothed using Gaussian kernel of $10 \mathrm{~mm}$.

Once the cortical models were complete, whole-brain cortical thickness was measured. It was calculated as the shortest distance between the GM/WM 
boundary and pial surface at each vertex across the cortex. The maps were created using spatial intensity gradients across tissue classes and were not simply reliant on absolute signal intensity. The maps produced are not restricted to the voxel resolution of the original data thus are capable of detecting submillimeter differences between groups. Subcortical volumes were also measured automatically using FreeSurfer by assigning each voxel in the normalized brain volume to one of 40 regions of interest (ROIs) (hippocampus, amygdala, thalamus, caudate, putamen, pallidum, among others) using a probabilistic atlas, from which we obtained the volume measurements. Total intracranial volume (TIV), was also calculated using Freesurfer and used to normalize volumetric data for the ROIs. To map all of the participants' brains to a common space and perform a comparison between groups, we registered all of the cortical thickness maps to a spherical atlas which is based on individual cortical folding patterns to match cortical geometry across participants (25) and create a variety of surface-based data.

\section{Cortical and subcortical statistical analysis}

The statistical analyses were carried out in two stages. First, entire cortex analyses were performed to explore cortical thickness in O-LOAD versus CS. Statistical maps were generated using the "command-line" group analysis stream in Freesurfer, which implements the General Linear Model (GLM) to estimate the differences in cortical morphometric data produced. The second stage consisted in performing the same analyses using age as a covariate. Even though intergroup age differences were non-significant, this was done due to the importance of age 
as a risk factor for LOAD. Multiple comparisons were corrected with a Monte Carlo Simulation using a two-tailed $p$-value set at $<0.05$. The results were visualized by overlaying significant cortical areas onto semi-inflated cortical surfaces.

Subcortical volumes were automatically derived from outcomes of Freesurfer, and SPSS 20.0 was used to analyze the differences in hippocampal volumes between groups. A significance level of $p<.05$ (Bonferroni multiple comparisons correction) was used.

\section{PET Image Acquisition}

\section{Synthesis of 18F-FDG: PET/ CT Equipment}

The PET image acquisition was performed on a PET/CT General Electric 690. Main FDG synthesis is executed in-house in a FASTLAB synthesizer. This synthesizer is a compact automated system that converts externally produced [18F]-fluoride into [18F]-FDG ready for quality control, usable in Positron Emission Tomography (PET). The synthesis is carried out in a disposable fluid pathway called FDG cassette. This cassette is replaced at each run, ensuring a clean and reproducible operation in every FDG production. The entire synthesis takes about 25 minutes. The resulting FDG solution (about $15 \mathrm{ml}$ ) and radionuclide is produced in the form of fluoride ions by bombardment of [180]-water $(2.5-5 \mathrm{ml})$ with accelerated protons on the cyclotron target. For in-house productions, the quantity of $18 \mathrm{~F}$-activity recovered is usually $1500-7000 \mathrm{mCi}$. During the irradiation, the 
synthesizer can carry out the cassette test and the synthesis preliminary steps (cassette conditioning). The participant must have been fasting for 6 hours, have a blood glucose level under $130 \mathrm{mg} / \mathrm{dl}$ and the procedure is conducted in a quiet dimlit room. Image acquisition was performed 30-40 min post- FDG administration, and dynamic tomographic images in 3D mode take approximately 30 min to acquire.

\section{Amyloid PET Imaging}

11C-PIB synthesis is carried out in a GE TRACER lab FXC PRO module which is a compact, automated radiochemistry system, which produces C-11 labeled radiochemicals from 11C-CO2. The module includes a HPLC (High Performance Liquid Chromatography) system for purification. The HPLC is a separation method that allows the isolation of the labeled product from radioactive by-products and organic impurities. In-house HPLC is performed with $0.009 \mathrm{M}$ sodium citrate ethanol/water (60/40) as a mobile phase with a flow rate of $3 \mathrm{~mL} / \mathrm{min}$ and a reverse phase high performance liquid chromatographic (RP-HPLC) column.

Chromatograms were registered using a UV-detector and a radioactivity detector in series. The product peak containing the [11C]-PIB is cut from the chromatographic system by valve switching from waste to product line. The [11C] PIB fraction (retention time 11-12 minutes) is transferred into flask and diluted with $30 \mathrm{~mL}$ of saline solution to reduce the ethanol percentage. The final total volume is $36 \mathrm{ml}$. The PIB solution is ready to be fractionated after sterile filtration. The $10 \mathrm{mCi}$ (adjusted by weight) was administered and image acquisition proceeded 50 min 
Post- 11CPIB administration. Subsequently, dynamic tomographic images, 3D mode takes 20 minutes.

\section{PET PiB and FDG Image Processing}

PET images were processed along with MRI volumetric T1 images. MRI T1 images were obtained and analyzed in FreeSurfer as described in the T1 Image

Processing section. Next, an analysis was performed using the PETSurfer scripts, which can be found at (https://surfer.nmr.mgh.harvard.edu/PetSurfer) and consist of the following steps: 1) creation of a high-resolution segmentation (gtmseg.mgz) used to run the Partial volume correction (PVC) methods for limited tissue sampling (26), 2) co-registration of PET and structural T1, 3) application of PVC method from which the PVC uptake of each region relative to Pons is obtained and volumes of corrected voxel-wise values of cortical and subcortical, using the "extended" Muller-Gartner method (we chose the pons because it is the region most commonly used in the type of analysis employed herein $(27,28,29,30))$, is easy to identify, has small PVEs, and is thought to be resistant to atrophy, at least in $A D(31)$, and 4) a surface-based analysis was carried out to sample those volumes onto each of the individual subject's surfaces.

\section{PET Statistical Analysis}


We applied the same two-stage procedure as described for cortical and subcortical statistical analyses. In this case the observed data for the GLM were the PIB or FGD volumes obtained from the step 3 .

\section{APOE genotyping}

Genomic DNA was obtained from peripheral blood leukocytes using the Wizard Genomic DNA Purification Kit (PROMEGA, Madison, WI, USA) according to manufacturer's instructions. One hundred micrograms of DNA were used as template in a PCR reaction using standard cycling conditions. PCR amplicons were digested with Cfol. Polyacrylamide gel electrophoresis followed by ethidium bromide staining was performed. Restriction fragments were analyzed upon observation at a UV transilluminator (32).

\section{General statistical procedures}

Intergroup comparisons were performed with an independent-samples t test or a Fisher's Exact Test for continuous and categorical variables respectively. All tests were two-tailed and the significance level was set at $p<0.05$.

\section{Results}


Table 1 shows the characteristics of the sample. As previously described $(13,14)$, O-LOAD exhibit significantly decreased MMSE score and episodic memory ability even though all participants fall within normal limits for each neuropsychological test. Further, this difference between groups probably reflects an evident ceiling effect that constrains the variance of this variable. $A P O E \varepsilon 4$ carrier status was more frequent among O-LOAD participants albeit the difference between groups was not significant (Table 1).

Figure 1 shows the differences in cortical thickness between O-LOAD and CS. The O-LOAD group displayed significantly reduced cortical thickness in bilateral precuneus, bilateral parietal cortex, right cingulate cortex, and areas of left occipital, right inferior temporal, and bilateral frontal cortices (Figure 1), but this difference became non-significant when controlling for age. Hippocampal volume did not differ between groups.

Figure 2 shows the map of FDG-PET intensity in O-LOAD and CS. Overall cortical FDG-PET intensity relative to pons was greater in O-LOAD compared to CS. Statistically significant regional differences in brain metabolic activity are shown in Figure 3. These included bilateral precunei, posterior cingulate, and posterior parietal cortices, as well as right middle cingulate and right temporoparietal cortex. Some of these areas (i.e., bilateral precunei, posterior parietal cortex, posterior cignulate, and right middle cingulate cortex) show a significant overlap with regions displaying decreased cortical thickness in the volumetric assessment (see Figure 1). When age was entered as a co-variate, the difference became non-significant. Hippocampal metabolic activity was similar in both groups. 
Figure 4 shows PET-PiB intensity signal in O-LOAD and CS. Both groups showed significant PiB signal intensity in the bilateral cingulate cortices, as well as in discrete areas of the temporal, frontal, insular, and parietal cortices. PiB intensity appears more extended in O-LOAD (left panels), but differences were not statistically significant when correcting for multiple measurements (not shown). However, when corrected for age, O-LOAD displayed greater PET-PiB signal than CS in the right hemisphere including temporoparietal cortex, as well as the postcentral, fusiform, inferior, middle temporal, and lingual gyri (Figure 5). PiB signal in the hippocampus displayed similar intensity in both groups (not shown).

\section{Discussion}

The main findings of the present study are as follows: 1) asymptomatic O-LOAD exhibited decreased cortical thickness in several brain regions shown to be affected in clinical forms of LOAD as well as in groups rendered at-risk for this disorder, from infants to the elderly $(33,34) ; 2)$ contrary to expectation, O-LOAD as a group exhibited greater brain metabolism in several regions, including some ADrelevant regions that evidenced significantly decreased thickness in this sample and 3) when controlling for age, PET-PIB signal was significantly more intense in the O-LOAD group compared with persons without family history of LOAD or other neurodegenerative illness in the right hemisphere, including areas shown to be affected in AD, but metabolic and structural intergroup differences were rendered non-significant. Pathophysiological models of $A D$ have proposed a sequence of 
events -and their corresponding biomarker changes- consisting of extracellular amyloid deposition, then intracellular neurofibrillary changes, and eventually neurodegeneration with varying degrees of neuroinflammation $(3,35)$. Most studies in LOAD have been conducted during symptomatic stages (i.e., amnestic mild cognitive impairment and Alzheimer's dementia) compared with normal individuals in advanced age, and have reported correlations between neurodegenerative and cognitive changes, and to a much lesser extent, between the latter and biomarkers of the severity of brain amyloid accumulation. Thus, it has been proposed that amyloid deposition preceded neurodegeneration and cognitive symptoms, either linearly over several years, or exponentially at an as yet undefined point in the natural history of the disorder, and then linearly (35). At the time of symptom onset, the amyloid deposit would, in theory, have already plateaued, thus explaining the relative dissociation between progression of amyloid accumulation and progression of cognitive symptoms (35). However, there is a paucity of studies specifically addressing AD-related structural and functional changes years prior to the expected onset of symptoms in LOAD, which is the most common form of the disorder. In a series of pioneering studies on the topic, Mosconi et al. $(36,8)$ found abnormalities in amyloid deposition, cerebral metabolism, and grey matter, in a sample of similar, albeit somewhat older participants, as ours (8). As expected, they detected increased amyloid and decreased cerebral glucose metabolism, the latter in AD-relevant areas, supporting the $\mathrm{ACH}$ prediction that amyloid deposition would precede other abnormalities at an early enough stage. Interestingly, they observed that maternal inheritance of LOAD was associated with these disturbances, whereas paternal inheritance was not $(36,8)$, even though lack of 
paternal inheritance of risk is not a uniform finding in the LOAD literature (6). Importantly, these observations were independent of the APOE genotype, which is the main known genetic risk factor for LOAD $(8)$. Reiman et al. $(12,11)$ in contrast, characterized the phenotypical consequences of the "dose" of ApoE4 in asymptomatic individuals (11). These authors did not take into account maternal vs paternal inheritance, and in one case, APOE $\varepsilon 4$ dose (i.e., homozygous, heterozygous, or no $A P O E \varepsilon 4$ allele) was examined among persons with at least one first-degree relative affected with the disorder, without comparison with individuals without family history (11). These authors concluded that the "dose" of $A P O E \varepsilon 4$ alleles was related to the intensity of in-vivo amyloid deposition and to decreased cerebral metabolism. Taken together, these studies provide support to the $\mathrm{ACH}$ in different groups of relatively young, asymptomatic individuals at epidemiological risk for LOAD, indicating both amyloid deposits and deficits in cerebral metabolism in AD-related areas are consistent and very early manifestations of risk for LOAD.

The present study partially replicates these observations, showing greater in vivo amyloid deposition in O-LOAD, when age is controlled for in intergroup comparisons. Apart from indications that amyloid deposition is associated with specific patterns of brain metabolic activity in young adults, LOAD patients, and cognitively normal older adults (37), different groups have also observed a positive correlation between cerebral metabolism and in vivo amyloid deposition as measured by $\mathrm{PiB}$ retention, and an inverse correlation of cerebral metabolism with cortical thickness $(38,39)$. Our findings are in agreement with the latter 
observations. Whereas increased metabolic activity in areas associated with AD (in which we found cortical thinning) could represent a compensatory response in areas that are adversely affected before the deposition of amyloid, our observation might be capturing an early phenomenon in which increased metabolism in ADrelevant areas favors increased deposition of amyloid $(37,39)$, and this might be occurring during a specific moment of neurodegeneration that seems very sensitive to subtle age changes.

We also observed consistent decrease of grey matter volume in AD-related regions such as the bilateral posterior cingulate cortex, precunei, right middle cingulate gyrus, and temporoparietal cortex prior to any significant mesial temporal neurodegeneration, which is the most consistent clinical neurodegenerative biomarker in LOAD (40), although the observed changes are likely age-dependent. This finding is intriguing as well, but a number of reasons could account for it. For one, different authors have found atrophy in these regions among at-risk individuals (33), sometimes in age groups so young that suggest that neurodegeneration is highly unlikely (e.g., infants who bear $A P O E \varepsilon 4$ genotypes; 34). These findings have raised the possibility that LOAD may be characterized by neurodevelopmental phenotypes in addition to, neurodegenerative phenotypes. Our results are consistent with this observation in that 1) neocortical thinning in AD-relevant areas was detected at a time without any evidence of hippocampal atrophy -a known early biomarker of neurodegeneration in clinical $A D$ and, 2) brain metabolic abnormalities in those same regions, which is similar to early findings in autosomal-dominant early-onset AD (41), but in contrast with how uptake is 
observed in AD during clinical stages. Moreover,, several genetic studies now suggest that gene variants associated to AD in old age, are also determinants of clinical (e.g., academic performance) and structural phenotypes (decreased cortical thickness in the same neocortical areas as in LOAD). These observations broaden the concept of LOAD from a purely neurodegenerative disorder, to include neurodevelopmental factors as well. This might also be a potential explanation for differences of MMSE and verbal learning in our sample. If this is true, the precise definition of the latter aspect might enable very early detection of future LOAD cases and, possibly, permit the design of very early intervention strategies.

Our conclusions are limited by a series of factors. Not being a prospective study, the differences in neuroimaging variables we observed between groups are of uncertain origin; moreover, we cannot make a definitive assessment of whether the differences observed in O-LOAD actually represent any increased risk of developing the disease. Our sample was homogeneous in regards to ethnicity, geographical area, culture, and years of education, perhaps limiting generalizability of the results. Also, the relatively small sample size might have obscured significant differences between groups, especially in regards to PET-PiB signal, and also prevented us from examining the effects of ApoE genotype and type of parental inheritance on the present results. Nonetheless, the current results have significant implications for understanding the earliest pathogenesis of LOAD and is worthy of further research.

In conclusion, in multimodal imaging of clinically asymptomatic individuals with at least one parent affected by LOAD, we observed cortical thinning in AD-relevant 
areas, and an unexpected increase of metabolic activity in those same areas. In this group, hippocampal volume and brain metabolic activity -compromised early in clinical forms of AD- were not affected. Amyloid deposition was greater in O-LOAD when we controlled for age. These changes seem very sensitive to minor, statistically non-significant age differences, thus underscoring that our sample might be capturing very early AD-related changes on neuroimaging. These series of observations are not entirely in line with the prevalent $\mathrm{ACH}$. In light of previous studies of both late- and early-onset $A D$ and at-risk subjects, we propose that a possible explanation for the discrepancy between what was expected and what was actually observed (especially increased metabolism in areas of decreased thickness), might be due to the fact that abnormalities described herein reflect both neurodevelopmental and early neurodegenerative AD-related phenotypes. The clarification of the sequence of events leading to LOAD is critical to help guide therapeutic measures, but would necessitate the study of a sufficient number of atrisk subjects of diverse age ranges, followed by longitudinal observation of their neuroimaging phenotypes. 


\section{Author Contributions}

MFV, DL, CBN, and SMG developed the scientific project. BDA and SV conducted the PET experiments. BDA, MFV, MNC, and SMS obtained and processed MRI images. BDA, SMS, MNC, and MFV analyzed the images. CA performed clinical and neuropsychological evaluations. CA, DV, RC, and DL processed and analyzed neuropsychological data. ES and GS processed and obtained genetic data. BDA, MFV, GS, CA, DL, CBN, RC, and SMG analyzed the results. BDA, MFV and SMG wrote the first draft of the manuscript. All authors edited and approved the final manuscript. 


\section{Role of funding source}

The present study was supported by a grant from Agencia de Promoción, FONCYT, MINCYT, Argentina (PICT-2014-0633). 


\section{Conflicts of interest}

Dr. Charles B. Nemeroff

Research/Grants:

National Institutes of Health (NIH), Stanley Medical Research Institute

Consulting (last three years):

Xhale, Takeda, Taisho Pharmaceutical Inc., Prismic Pharmaceuticals, Bracket

(Clintara), Total Pain Solutions (TPS), Gerson Lehrman Group (GLG) Healthcare \&

Biomedical Council, Fortress Biotech, Sunovion Pharmaceuticals Inc., Sumitomo

Dainippon Pharma, Janssen Research \& Development LLC, Magstim, Inc., Navitor

Pharmaceuticals, Inc., TC MSO, Inc., Intra-Cellular Therapies, Inc.

Stockholder:

Xhale, Celgene, Seattle Genetics, Abbvie, OPKO Health, Inc., Network Life

Sciences Inc., Antares, BI Gen Holdings, Inc.

Scientific Advisory Boards:

American Foundation for Suicide Prevention (AFSP), Brain and Behavior Research

Foundation (BBRF) (formerly named National Alliance for Research on

Schizophrenia and Depression [NARSAD]), Xhale, Anxiety Disorders Association

of America (ADAA), Skyland Trail, Bracket (Clintara), RiverMend Health LLC,

Laureate Institute for Brain Research, Inc.

Board of Directors:

AFSP, Gratitude America, ADAA

Income sources or equity of $\$ 10,000$ or more:

American Psychiatric Publishing, Xhale, Bracket (Clintara), CME Outfitters, Takeda 
Patents:

Method and devices for transdermal delivery of lithium (US 6,375,990B1)

Method of assessing antidepressant drug therapy via transport inhibition of

monoamine neurotransmitters by ex vivo assay (US 7,148,027B2)

Speakers Bureau:

None

All other authors have no disclosures. 


\section{Acknowledgments}

BDA is a doctoral fellow from Agencia. SDG. CA and SMS are doctoral fellows from CONICET.

\section{References}

1.Alzheimer, A., Über eine eigenartige Erkrankung der Hirnrinde. Vortrag in der Versammlung Südwestdeutscher Irrenärzte in Tübingen am 3. November 1906. Allg Z Psychiatr Psych Gerichtl Med, 1907. p.146-148

2.Kraepelin, E., Psychiatrie. Ein Lehrbuch für Studierende und Ärzte. Achte, vollständigumge arbeitete Auflage. II. Band. Klinische Psychiatrie. I.Teil. Barth Verlag, Leipzig 1909.

3.Hardy, J.A., Higgins, G.A., Alzheimer's disease: the amyloid cascade hypothesis. Science, 1992. 256(5054): p.184-5

4.Cummings, J.L., Morstorf, T., Zhong, K., Alzheimer's disease drug-development pipeline: few candidates, frequent failures. Alzheimers Res Ther, 2014. 6(4): p.37

5.Herrup, K., The case for rejecting the amyloid cascade hypothesis. Nat Neurosci, 2015. 18(6): p.794-9

6.Bertram, L., Lill, C.M., Tanzi, R.E., The genetics of Alzheimer disease: back to the future. Neuron, 2010. 68(2): p.270-81 
7. Mosconi, L., Berti, V., Swerdlow, R.H., et al., Maternal transmission of Alzheimer's disease: prodromal metabolic phenotype and the search for genes. Human Genomics, 2010. 4(3): p.170-93

8.Mosconi, L., Murray, J., Tsui, W.H., et al., Brain imagine of cognitively normal individuals with 2 parents affected by late-onset AD. Neurology, 2015. 82(9): p.752-760

9.During, E.H., Osorio, R.S., Elahi, F.M., et al., The concept of FDG-PET endophenotype in Alzheimer's disease. Neurol Sci, 2011. 32(4): p.559-569 10.Mosconi, L., Rinne, J.O., Tsui, W.H., et al., Amyloid and metabolic positron emission tomography imaging of cognitively normal adults with Alzheimer's parents. Neurobiol Aging, 2013. 34(1): p.22-34

11.Reiman, E.M., Chen, K., Alexander, G.E., et al., Correlations between apolipoprotein E $\varepsilon 4$ gene dose and brain-imaging measurements of regional hypometabolism. Proc Natl Acad Sci USA, 2005. 102(23): p.8299-8302

12.Reiman, E.C., Caselli, R.J., Chen, K., et al., Declining brain activity in cognitively normal apolipoprotein $E \varepsilon 4$ heterozygotes: a foundation for using positron emission tomography to efficiently test treatments to prevent Alzheimer's disease. PNAS, 2001. 98(6): p.3334-3339

13.Abulafia, C., Duarte-Abritta, B., Villarreal, M.F., et al., Relationship between Cognitive and Sleep-wake Variables in Asymptomatic Offspring of Patients with Late-onset Alzheimer's Disease. Front Aging Neurosci, 2017. 9: p.93 
14.Sánchez, S.M., Abulafia, C., Duarte-Abritta, B., et al., Failure to Recover from Proactive Semantic Interference and Abnormal Limbic Connectivity in Asymptomatic, Middle-Aged Offspring of Patients with Late-Onset Alzheimer's Disease. J Alzheimer Dis, 2017. 60(3): p.1183-1193

15.Albanese, E., Dangour, A.D., Uauy, R., et al., Dietary fish and meat intake and dementia in Latin America, China, and India: a 10/66 Dementia Research Group population-based study. Am J Clin Nutr, 2009. 90(2): p.392-400

16.Segonne, F., Dale, A.M., Busa, E., et al., A hybrid approach to the skull stripping problem in MRI. Neuroimage, 2004. 22(3): p.1060-1075

17.Fischl, B., Salat, D.H., Busa, E., et al., Whole brain segmentation: automated labeling of neuroanatomical structures in the human brain. Neuron, 2002. 33(3): p.341-355

18.Fischl, B., Salat, D.H., van der Kouwe, A.J., et al., Sequence-independent segmentation of magnetic resonance images. Neuroimage, 2004a. 23 Suppl 1: S69-84

19.Sled, J.G., Zijdenbos, A.P., Evans, A.C., A nonparametric method for automatic correction of intensity nonuniformity in MRI data. IEEE Trans Med Imaging, 1998. 17(1): p.87-97

20.Fischl, B., Liu, A., Dale, A.M., Automated manifold surgery: constructing geometrically accurate and topologically correct models of the human cerebral cortex. IEEE Trans Med Imaging, 2001. 20(1): p.70-80 
21.Segonne, F., Pacheco, J., Fischl, B., Geometrically accurate topologycorrection of cortical surfaces using nonseparating loops. IEEE Trans Med Imaging, 2007. 26(4): p.518-29

22.Dale, A.M., Fischl, B., Sereno, M.I., Cortical surface-based analysis. I. Segmentation and surface reconstruction. Neuroimage, 1999. 9(2): p.179-94

23.Dale, A.M., Sereno, M.I., Improved localization of cortical activity by combining EEG and MEG with MRI cortical surface reconstruction: a linear approach. J Cogn Neurosci, 1993. 5(2): p.162-76

24.Fischl, B., Dale, A.M., Measuring the thickness of the human cerebral cortex from magnetic resonance images. Proc Natl Acad Sci U S A, 2000. 97(20): p. $11050-5$

25.Fischl, B., Sereno, M.I., Tootell, R.B., et al., High-resolution intersubject averaging and a coordinate system for the cortical surface. Hum Brain Mapp, 1999b. 8(4): p.272-84

26.Greve, D.N., Salat, D.H., Bowen, S.L., et al., Different partial volume correction methods lead to different conclusions: An 18 F-FDG-PET study of aging. Neurolmage, 2016. 132: p.334-343

27. Ewers, M., Brendel, M., Rizk-Jackson, et al., Alzheimer's Disease Neuroimaging I. Reduced FDG-PET brain metabolism and executive function predict clinical progression in elderly healthy subjects. Neuroimage Clin, 2014. 4: p.45-52. 
28. Kantarci, K., Jack, C.R. Jr., Xu, Y.C., et al., Mild cognitive impairment and Alzheimer disease: regional diffusivity of water. Radiology, 2001. 219(1): p101107.

29. Knopman, D.S., Jack, C.R., Jr. Wiste, H.J., et al., 18F-fluorodeoxyglucose positron emission tomography, aging, and apolipoprotein E genotype in cognitively normal persons. Neurobiol Aging, 2014. 35(9): p.2096-2106.

30. Landau, S.M., Harvey, D., Madison, C.M., et al., Alzheimer's Disease Neuroimaging I. Associations between cognitive, functional, and FDGPET measures of decline in $A D$ and MCl. Neurobiol Aging, 2011. 32(7): p.1207-1218.

31. Minoshima, S., Frey, K.A., Foster, N.L., et al., Preserved pontine glucose metabolism in Alzheimer disease: a reference region for functional brain image (PET) analysis. J Comput Assist Tomogr, 1995. 19(4): p.541-547.

32.Hixson, J.E., Vernier, D.T., Restriction isotyping of human apolipoprotein E by gene amplification and cleavage with Hhal. J Lipid Res, 1990. 31(3): p.545-8

33. Reiter, K., Alpert, K.I., Cobia, D.J., et al., Cognitively normal individuals with $A D$ parents may be at risk for developing aging-related cortical thinning patterns characteristic of $A D$. Neuroimage, 2012. 61(3): p.525-532 
34.Dean, D.C. $3^{\text {rd }}$, Jerskey, B.A., Chen, K., et al., Brain differences in infants at differential genetic risk for late-onset Alzheimer disease: a cross-sectional imaging study. JAMA Neurol, 2014. 71(1): p.11-22

35.Jack, C.R. Jr, Lowe, V.J., Weigand, S.D., et al., Serial PIB and MRI in normal, mild cognitive impairment, and Alzheimer's disease: implications for sequence of pathological events in Alzheimer's disease. Brain, 2009. 132(Pt5): p.1355-1365

36.Mosconi, L., de Leon M, Murray, J., et al., Reduced mitochondria cytochrome oxidase activity in adult children of mothers with Alzheimer's disease. J Alzheimers Dis, 2011. 27(3): p.483-90

37.Arnemann, K.L, Stöber, F., Narayan, S., et al., Metabolic Brain networks in aging and preclinical Alzheimer`s disease. Neuroimage Clin, 2018. 17: p.987-999

38. Oh, H., Habeck, C., Madison, C., et al., Covarying alterations in A $\beta$ deposition, glucose metabolism, and gray matter volumen in cognitively normal elderly. Hum Brain Mapp, 2014. 35(1): p.10.

39.Cohen, A.D., Prince, J.C., Weissfeld, L.A., et al., Basal cerebral metabolism may modulate the cognitive effects of $A \beta$ in Mild Cognitive Impairment: An example of brain reserve. J Neurosci, 2009. 29(47): p.14770.

40.Dubois, B., Hampel, H., Feldman, H.H., et al., Preclinical Alzheimer's disease: definition, natural history, and diagnostic criteria. Alzheimer's \& Dementia, 2016. 12(3): p.292-323 
41.Benzinger, T.L.S., Blazey, T., Jack, C.R. Jr., et al., Regional variability of imaging biomarkers in autosomal dominant Alzheimer's disease. Proc Natl Acad Sci USA, 2013. 110(47): E4502-9 
Table 1. Demographic data

\begin{tabular}{|c|c|c|c|c|c|c|}
\hline & \multicolumn{6}{|c|}{ Group } \\
\hline & \multicolumn{2}{|c|}{$\operatorname{CS}(n=25)$} & \multicolumn{2}{|c|}{ O-LOAD $(n=30)$} & \multirow[t]{2}{*}{ Statistic } & \multirow[t]{2}{*}{$\mathrm{p}$} \\
\hline & $\begin{array}{l}\text { Mean or } \\
\text { Frequency }\end{array}$ & $\mathrm{SD}$ or $\%$ & $\begin{array}{l}\text { Mean or } \\
\text { Frequency }\end{array}$ & SD or $\%$ & & \\
\hline Female & 21 & $84 \%$ & 20 & $66.7 \%$ & $X^{2}=2.159$ & .142 \\
\hline Maternal Inheritance & $\mathrm{N} / \mathrm{A}$ & $\mathrm{N} / \mathrm{A}$ & 16 & $53.3 \%$ & & \\
\hline Age & 51 & 7.6 & 54.4 & 7 & $t=-1.724$ & .091 \\
\hline Education & 17.6 & 2.7 & 17.4 & 3 & $\mathrm{t}=.238$ & .813 \\
\hline APOE $\varepsilon 4$ & 1 & $8.3 \%$ & 4 & $23.5 \%$ & $X^{2}=1.138$ & .286 \\
\hline WAT-BA & 43.8 & 4.9 & 41.9 & 5.6 & $t=1.373$ & .175 \\
\hline BDI II & 8.1 & 7.5 & 8.9 & 6.5 & $t=-.421$ & .676 \\
\hline MMSE & 29.6 & 0.7 & 28.8 & 1.2 & $t=3.046$ & .004 \\
\hline RAVLT Delayed R & 10.3 & 2 & 8.5 & 3 & $t=2.647$ & .011 \\
\hline
\end{tabular}

BDI-II: Beck Depression Inventory, second edition; WAT-BA: Word Accentuation Test, Buenos Aires version; MMSE: Mini Mental State Examination; RAVLT Delayed R: Rey Auditory Verbal Learning Test delayed recall. 


\section{Figure Legends}

Figure 1: Differences in cortical thickness between offspring of patients with lateonset Alzheimer's disease (O-LOAD) and comparable individuals without family history of neurodegenerative disorders (CS). The clusters in red indicate greater

thickness in CS vs O-LOAD, the values of the maps shown correspond to $-\log (p)$ with $p<0.05$, following Monte Carlo correction.

Figure 2: FDG-PET intensity maps in O-LOAD and CS. The colors indicate the intensity of, greater FDG $(-\log (p))$ intensity values are shown in yellow and lower values in red.

Figure 3: Differences in regional brain metabolism as measured by FDG-PET between O-LOAD and CS. Blue areas indicate regions of lower metabolic activity in CS. No brain region shows greater metabolic activity in CS compared to OLOAD. Please see the text for details.

Figure 4: PET-PiB intensity maps in O-LOAD and CS. The colors indicate the intensity of $\mathrm{PiB}(-\mathrm{lo}(\mathrm{p}))$, with higher values in yellow and lower values in red.

Figure 5: Differences in regional brain $\beta$-amyloid deposit as measured by PET-PiB, using age as a covariate between O-LOAD and CS. Values correspond to -log ( $p)$ corrected by Monte Carlo. Blue areas indicate greater PET-PiB signal in O-LOAD. 
Figure 1.

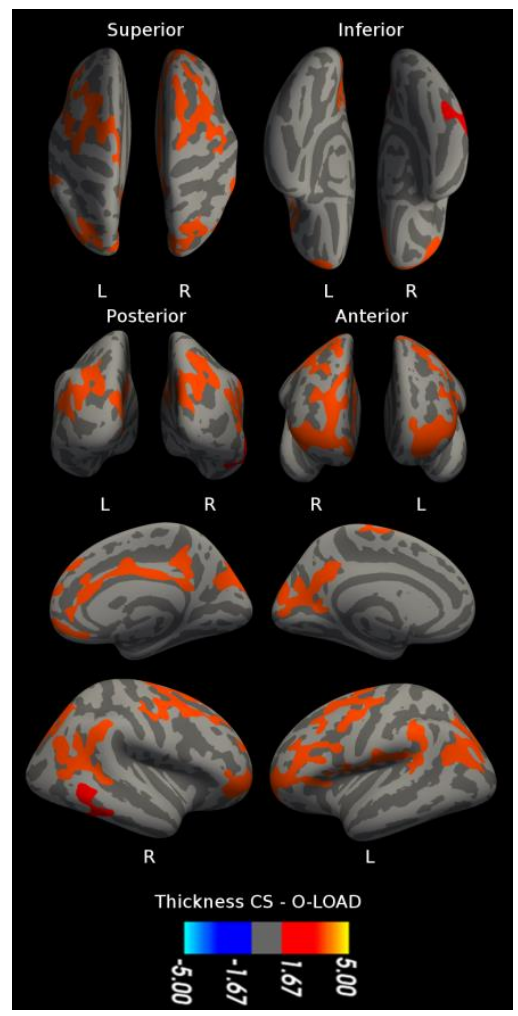


Figure 2.

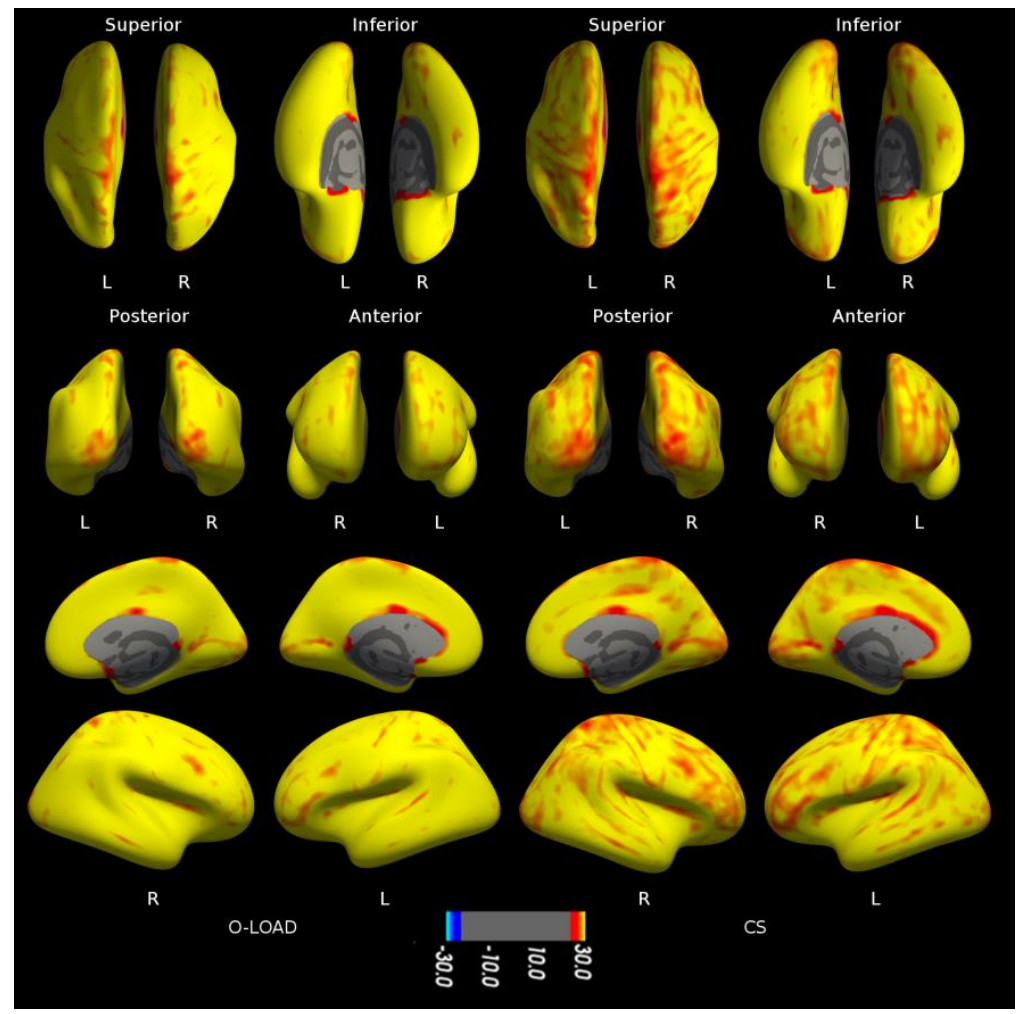


Figure 3.

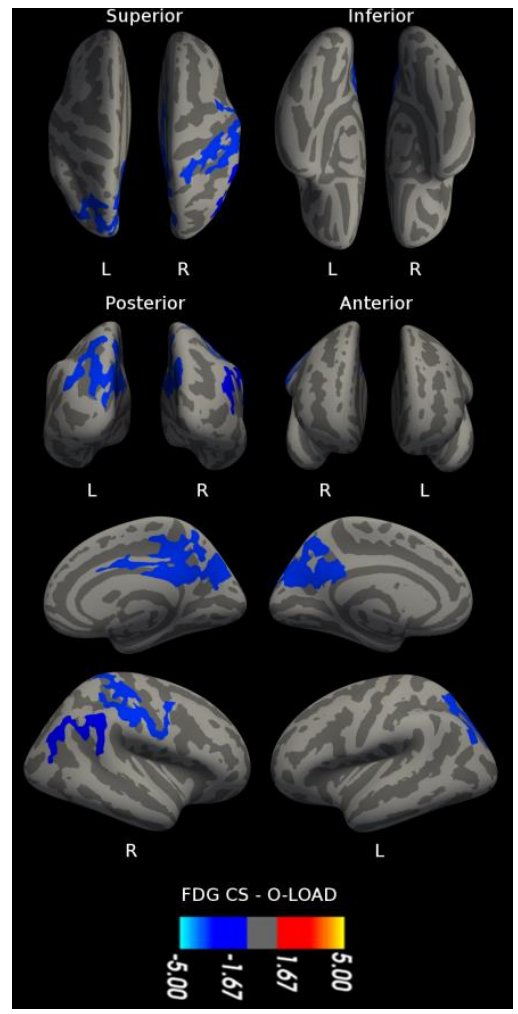


Figure 4.

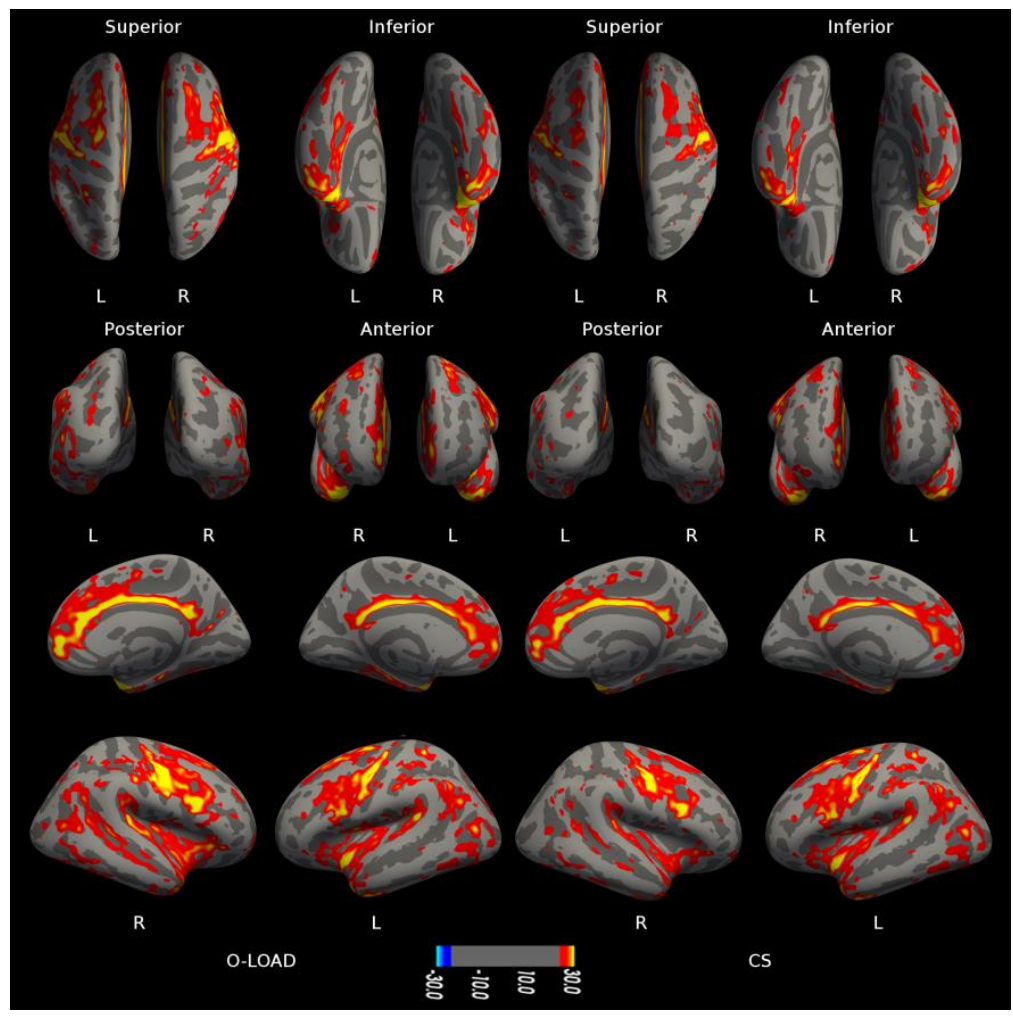


Figure 5.

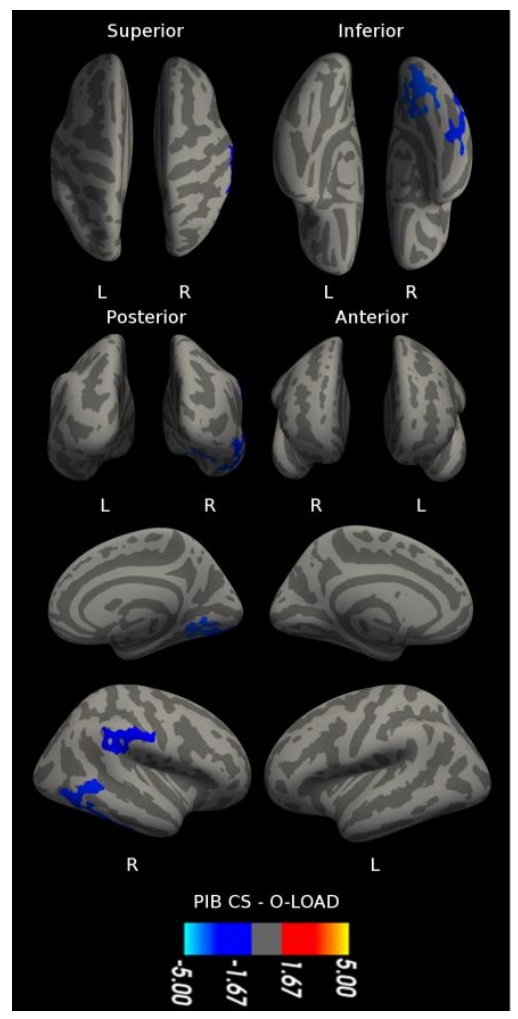

